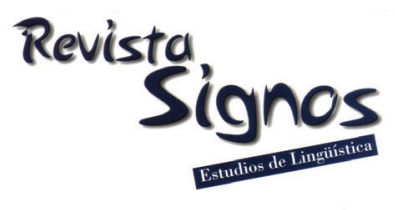

\title{
Géneros en la enseñanza escolar: Configuraciones de significado en clases de historia y biología desde una perspectiva multimodal
}

\section{Genres in school teaching: Configurations of meaning in History and Biology lessons from a multimodal perspective}

\author{
Dominique Manghi \\ Pontificia Universidad Católica de Valparaíso \\ CHILE \\ dominique.manghi@ucv.cl
}

Recibido: 30-VII-2012 / Aceptado: 4-I-2013

\section{Resumen}

Los géneros como configuraciones de significado con un propósito social (Martin \& Rose, 2008a) en el contexto de las clases en Educación Media responden tanto a las necesidades de la enseñanza como a las especificidades de la disciplina que recontextualizan y resemiotizan los profesores en las aulas. En este sentido, tanto los profesores de biología como los de historia despliegan configuraciones de significado construidas a partir de combinaciones semióticas en función tanto de propósitos de enseñanza, así como de las condiciones sociales y materiales del contexto del aula. Este estudio describe desde la perspectiva multimodal los principales géneros para la enseñanza identificados en clases de biología y de historia en primer año Educación Media. El corpus audiovisual está conformado por las grabaciones de las clases de tres profesores de biología y tres de historia de primer año de Educación Media de colegios particulares subvencionados de la $\mathrm{V}$ región, Chile. Los hallazgos nos indican que para la historia los relatos históricos así como las explicaciones factoriales se despliegan con una combinación semiótica prototípica. Mientras que en clases de biología tanto las definiciones -descriptivas, composicionales y clasificaciones- como las explicaciones secuenciales son construidas por los profesores a partir de una orquestación multimodal. Mediante estos géneros y sus combinaciones semióticas, los profesores acercan a los estudiantes a las formas de pensar y comunicar propias de cada disciplina.

Palabras Clave: Géneros discursivos, discurso pedagógico, semiótica social, multimodalidad, mediación semiótica. 


\begin{abstract}
Genre as a configuration of meaning with a social purpose (Martin \& Rose, 2008a) in the context of secondary school meets both the needs of teaching and the specificities of the discipline resemiotized and recontextualized by teachers in their lessons. In this sense, both history and biology teachers unfold meaning configurations constructed upon semiotic combinations according to teaching purposes as well as social and material conditions of the classroom context. This study describes, from a multimodal perspective, the main genres for teaching identified in biology and history in the first year of secondary school. The corpus consists of audio-visual recordings of the lessons of three teachers of each course in first year of secondary school in Valparaíso Region, Chile. The findings show that historical accounts and factorial explanations are unfold in History lessons through prototypical semiotic combinations. While in Biology lessons, definitions -descriptions, classifications, compositions- and sequential explanations are built by teachers on a multimodal orchestration. Through these genres and their semiotic combinations, teachers get their students closer to the ways of thinking and communicating specific to each discipline.
\end{abstract}

Key Words: Genres, pedagogic discourse, social semiotics, multimodality, semiotic mediation.

\title{
INTRODUCCIÓN
}

En los últimos años, la lingüística ha descrito la lengua en uso y uno de los contextos en los que ha puesto atención es el educativo. Algunos investigadores se han aproximado a este problema desde el estudio de los géneros que son utilizados para enseñar en diferentes niveles de la educación. A nivel nacional, los estudios han caracterizado el discurso de la historia desde el análisis crítico describiendo los recursos lexicogramaticales utilizados en los manuales de historia usados en los colegios para construir el pasado reciente (Oteíza, 2006; Oteíza \& Pinto, 2011); también la lengua escrita a través de la lingüística de corpus, identificando las coocurrencias léxicas y gramaticales en los manuales de estudio en educación técnico profesional (Parodi, 2010). Otras propuestas en Latinoamérica han abordado también los textos o manuales escolares argentinos (Moyano, 2010) y colombianos (Moss \& Chamorro, 2011) con propuestas de alfabetización académica en trabajos con los profesores de aula.

Junto a estas líneas de investigación, encontramos otra que se ha centrado en la lengua oral usada en las aulas. Es así como se han descrito los distintos géneros que emergen de las interacciones de aula mediante los cuales los profesores trabajan los textos escritos (Barletta \& Mizuno, 2011).

Todos estos estudios dan cuenta de la importancia de describir cómo es que los profesores enseñan a sus aprendices conocimientos que se alejan del sentido común 
y que requieren de una mediación para su aprendizaje, para comprender mejor cómo es actualmente y cómo avanzar hacia lo que debería ser la practica pedagógica. La perspectiva de la Lingüística Sistémica Funcional pone su foco en que el discurso de la enseñanza no apunta solamente a lograr que los estudiantes memoricen las fechas y personajes de la historia, o las taxonomías y nombres técnicos de la biología, sino más bien aprendan cierta forma particular de interpretar que está lejos de lo cotidiano y que implica usar los recursos discursivos para representar, comunicar y valorar ese conocimiento de una manera especial (Halliday \& Martin, 1993; Christie \& Derewianka, 2010; Martin, Maton \& Matruglio, 2010).

La presente investigación plantea la descripción del discurso de los profesores desde dos perspectivas diferentes. Primero, se propone describir los géneros que utilizan los profesores en la interacción cara a cara con fines de enseñanza para describir cómo es que se enseña esta forma particular de representar y comunicar en la disciplina. Y segundo, asume que, para poder observar de manera más completa la interacción áulica y los significados que allí se construyen, es necesario aproximarse al discurso de los profesores desde la perspectiva multimodal. Es decir, la descripción de los géneros que aquí se propone considera de manera importante cómo los recursos semióticos típicos del contexto escolar interaccionan en las configuraciones de significado para la enseñanza en la escuela. Los hallazgos de esta investigación aportan a la descripción del discurso oral, el discurso para la enseñanza, así como también la formación de profesores.

A continuación se presentan los fundamentos teóricos de este estudio, la metodología y los principales hallazgos en relación con los géneros utilizados por los profesores para la creación de significado con fines de enseñanza de la historia y de la biología.

\section{Marco teórico}

Los fundamentos teóricos que se presentan en este apartado sientan las bases para comprender qué observa un analista cuando describe el discurso de un profesor. Este estudio adopta la teoría de la Semiótica Social y la Lingüística Sistémica Funcional (en adelante LSF) para conceptualizar al profesor y su discurso en contexto educativos.

\subsection{Los significados en el discurso del profesor: Convenciones, contexto $y$ configuraciones}

En los estudios lingüísticos el discurso del profesor ha sido conceptualizado desde diferentes ángulos, en este estudio se concibe como una instancia de semiosis bastante particular en la que priman los propósitos pedagógicos para una recontextualización de discursos primarios, en este caso el discurso de la historia y el de la biología, en la cual es posible reconocer ciertos patrones de significado regulares que el profesor despliega para esa situación comunicativa específica. 
El profesor es un usuario de un sistema semiótico quien utiliza distintas opciones para significar, siempre en una determinada situación de comunicación (Halliday, 1982). De esta definición se pone el foco en tres ideas; la primera respecto de los sistemas semióticos y los usuarios; la segunda, referida a la relación entre los significados y el contexto así como su recontextualización; y la tercera, relacionada con las configuraciones de significado.

En relación con los sistemas semióticos, la teoría de la Semiótica Social propone que cada grupo social o comunidad ha estabilizado recursos para crear significados con el propósito de cubrir sus necesidades de comunicación y representación (Halliday, 1982; Hodge \& Kress, 1988; van Leeuwen, 2005). Cualquier sistema semiótico ofrece la posibilidad de construir dos tipos de significado simultáneamente: entablar relaciones sociales con otros (significado interpersonal) así como también representar la experiencia (significado ideacional). Junto con estas dos, existe una tercera función que es la de habilitar los dos significados anteriores textualizándolos (significado textual).

De esta manera, la comunidad de los historiadores ha convencionalizado ciertas formas de crear dichos significados o semiosis y, de la misma manera, la comunidad de los biólogos ha conformado su propia forma de significar. Cada uno de ellos corresponde a un discurso que los diferencia de los otros grupos sociales y que les permite crear y recrear el conocimiento.

Desde esta teoría, el discurso de los científicos (incluyendo las ciencias humanas y sociales), responde a la necesidad de reconstruir la realidad como un mundo de relaciones lógicas entre entidades abstractas (Halliday \& Martin, 1993). Existe un bagaje de investigaciones desde la LSF que ha profundizado en las particularidades de los distintos discursos científicos llevados al ámbito pedagógico, especialmente en contextos de lengua inglesa, tanto a nivel discursivo como lexicogramatical.

Centrándose en la lengua, en relación con los rasgos lexicogramaticales en el discurso de la enseñanza de la historia, autores como Coffin (1997); Martin (2003); Martin y Rose (2008a), y en contextos latinoamericanos: Oteíza (2006); Moyano (2010); Giudice y Moyano (2011) han descrito las opciones semióticas para enseñar historia. Sus investigaciones dan cuenta de rasgos de este discurso como la impersonalidad, la nominalización, la infinitivización así como el uso de procesos existenciales y relacionales en clausulas declarativas para construir eventos conflictivos de manera objetiva.

En cuanto al discurso de la biología en contextos de enseñanza, autores como Halliday y Martin (1993); Márquez, Izquierdo y Espinet (2000); Moyano (2010); Moss y Chamorro (2011), nos indican que el discurso pedagógico de la biología hace uso de diferentes recursos semióticos para construir la experiencia a través de un discurso donde el universo se representa inmutable, lejos del aquí y ahora, y alejado también 
de la acción humana, a través de taxonomías analíticas (partes-todo) y clasificatorias muy detalladas y rígidas. Los conocimientos se representan de manera impersonal sin agentes ni responsables de las teorías, construyendo así una representación objetiva de la ciencia, a través de nominalizaciones y un alto nivel de abstracción que impacta en la organización textual, y en las relaciones lógicas implícitas o construidas como metáfora lógica (Moyano, 2010).

Desde la sociología de la educación, la diferencia entre ambos discursos radica principalmente en la naturaleza del conocimiento acumulado que cada una de estas disciplinas ha construido (Bernstein, 2000; Maton, 2007). De esta manera, la historia corresponde a una estructura de conocimiento horizontal, por lo que la descripción ideacional o de la experiencia tal como es vista desde la historia, es representada a través de conceptos abstractos y términos utilizados de manera especializada, los que se despliegan en diferentes definiciones paralelas (Martin et al., 2010). La biología, por otra parte, ha desarrollado una estructura de conocimiento vertical, por lo que sus significados ideacionales dan cuenta de taxonomías únicas que definen de manera muy precisa y detallada cada concepto, el cual a su vez puede volver a desplegar una nueva taxonomía en su interior (Halliday \& Martin, 1993).

Los rasgos mencionados para los discursos de la historia y de la biología, permiten la construcción de cada teoría científica como una construcción lingüística de la experiencia diferente de la que construye la gramática del discurso cotidiano. Esto nos indica que aprender a comunicarse como se hace en historia y en biología implica alejarse de un conocimiento y discurso de sentido común, para apropiarse de un conocimiento y discurso fuera del sentido común, una manera particular de entender el mundo desde las ciencias (Veel, 1998; Moss \& Chamorro, 2011). En esta forma científica de ver el mundo, la lengua posibilita clasificar y construir taxonomías en torno a estos significados, creando las teorías científicas (Lemke, 1998).

En cuanto a la segunda idea, la relación entre los significados y el contexto, el enfoque la LSF enfatiza los vínculos sistemáticos que existen entre la organización de una lengua -entendida como semiótica social- y la organización del contexto (Eggins \& Martin, 2003). El contexto de situación se describe en términos de tres variables que caracterizan distintos tipos de manifestaciones concretas de uso de la lengua distintos registros en términos de LSF-. Las tres variables son el tema y la actividad que se desarrolla (campo), la relación entre los interlocutores (tenor) y los distintos modos o recursos semióticos disponibles en esa situación (modo) (Halliday, 1982). En el caso de este estudio, el registro corresponde al escolar y más específicamente a clases de historia o clases de biología, en las cuales un profesor especialista se relaciona con estudiantes de primer año de Educación Media, utilizando los recursos semióticos propios de esta situación: lengua oral, pizarrón, cuaderno, entre otros.

En relación al contexto educativo, la sociología de la educación aporta un interesante aspecto a considerar para la definición del registro educativo y las 
opciones de los profesores para construir significados. Los registros de la historia y el de la biología como tal, pertenecen a sus contextos primarios, aquellos que se definen por las actividades propias de los historiadores y biólogos, y las relaciones sociales que mantienen en sus comunicaciones dentro de la comunidad. Sin embargo, el discurso del profesor no corresponde a este mismo registro ya que ha sufrido un cambio en un proceso en el que se distinguen dos etapas: una en el que se seleccionan ciertos aspectos de la historia y de la biología para ser parte del currículo nacional a ser enseñado en las escuelas; y una segunda etapa en la cual este discurso -que ya ha sido descontextualizado- es nuevamente recontextualizado a través del discurso de cada profesor en un aula. Este proceso corresponde al dispositivo pedagógico (Bernstein, 1990) a través del cual los discursos primarios se transforman en discursos pedagógicos. En este estudio se rescata esta idea, sin embargo, se amplía y agentiviza el rol de los actores del proceso social (Latour, 2008), desde un discurso que se recontextualiza y luego reproduce -en los términos de Bernstein- hacia un discurso que es descontexualizado por determinadas autoridades y bajo ciertos criterios para ser parte del currículo nacional, el que luego nuevamente es mediado por cada profesor de manera activa en cada recontextualización con fines de enseñanza.

En este proceso de recontextualización, es necesario considerar las condiciones del contexto que afectan las diferentes manifestaciones concretas o instancias del discurso de profesores. El principio básico para un análisis desde la Semiótica Social es que los seres humanos crean significado llevando a cabo una selección de acuerdo a los recursos disponibles así como al contexto situacional y de cultura en los que se encuentran (Malinowsky, 1933; Lemke, 1998).

Dicha perspectiva nos permite conceptualizar los discursos de la enseñanza y el aprendizaje en las clases escolares como evidencia de las opciones de profesores y estudiantes para crear significado a partir de los recursos disponibles en ese determinado contexto educativo y momento socio histórico (Jewitt, Kress, Ogborn \& Tsetarellis, 2001). Este es el concepto de resemiotización que propone Iedema (2003) para enfatizar en la relevancia de las condiciones sociales y materiales de cada re-contextualización -en términos de Bernstein (1990)- e instanciación - en términos de Halliday (1982).

La tercera idea que colabora en la definición del discurso del profesor tiene que ver con las configuraciones semánticas o géneros. Para Martin y Rose (2008a) los géneros se definen como procesos sociales orientados a un objetivo y organizados en etapas. Se consideran como práctica social ya que se participa en estas configuraciones de significado junto con otras personas, además se persiguen metas porque los géneros son utilizados para hacer cosas, y se requieren varios pasos para alcanzar el propósito retórico. Desde esta propuesta de género la función retórica es responsable de la organización estructural de los textos y es parte del modelo estratificado de textocontexto (Eggins \& Martin, 2003). 
El modelo estratificado de la LSF distingue dos continuos que confluyen para la concepción de género desde la propuesta de Martin. El primero de ellos es el continuo de la realización, en el cual el género constituye el grado máximo de abstracción, un patrón de patrones de significado a nivel de la cultura. Mientras que desde el segundo continuo, el de la instanciación o jerarquía de la generalización, el género es un paso intermedio entre el sistema completo como potencial de opciones para significar y los tipos de textos y tipos de instancias de interpretación de los mismos. En este segundo continuo, género y registro se encuentran a mismo nivel de generalidad. De esta manera, diferentes tipos de textos enactúan diversos tipos de contextos sociales (Martin \& Rose, 2008b).

Observar los géneros desde la jerarquía de la instanciación, permite interpretar la configuración de significado acotada a un registro particular. La noción de género releva la importancia de la secuenciación temporal de los significados, poniendo el foco en el despliegue de los significados en el tiempo o logogénesis en términos hallidayanos.

A partir de las ideas de género y de discurso pedagógico, Christie (2002) propone que en el contexto de la sala de clases se crean unidades semánticas particulares que se despliegan en la interacción del aula. En el discurso del registro escolar es posible establecer unidades de significado mayores que responden al despliegue de una unidad curricular completa como un diseño del profesor, las que pueden extenderse a lo largo de varias sesiones de clases. Dichas unidades de significado son denominadas por Christie (2002) macrogéneros curriculares, los que a su vez, están constituidos por cada una de las clases. Estas últimas pueden ser reconocidas como una unidad de significado en sí mismas, es decir, un género curricular.

Para poder comprender entonces el discurso del profesor y los significados que construye en interacción con sus estudiantes, resulta esencial considerar el valor de las secuencias de semiosis en función de la totalidad de la estructura de la actividad curricular, interpretando cada fragmento textual en función del ciclo completo, considerando los movimientos y el desarrollo del texto a través de la actividad de enseñanza desplegada en el tiempo.

\subsection{Discurso del profesor: Semiosis e intersemiosis en el aula}

El último aspecto teórico que es necesario apuntar, se relaciona con la semiosis en el aula escolar y la multimodalidad. Este estudio aborda la sala de clases poniendo atención en la diversidad de formas de representar y comunicar presentes en el panorama semiótico del aula.

El concepto de multimodalidad corresponde a una perspectiva para observar y conceptualizar la comunicación, ampliando el foco desde los significados construidos esencialmente mediante la lengua hacia la variedad de modos o recursos semióticos 
utilizados por los seres humanos para significar y que confluyen en un mismo evento comunicativo (O'Halloran \& Smith, 2011). Este enfoque pone de manifiesto que la lengua no es el único código que posibilita acceder a la construcción e intercambio de significados, sino que abarca diferentes convenciones y recursos semióticos que sirven a este propósito como esquemas, gráficos, fotos, dibujos, fórmulas, etc. Es por esto que la multimodalidad más que una teoría nueva, constituye un campo de aplicación o un enfoque sobre la representación y comunicación (O’Halloran \& Smith, 2011), el cual se nutre de otras teorías como la lingüística (Jewitt, 2008) y a la vez puede enriquecerlas.

Desde esta perspectiva, cualquier texto que significa a través de la interacción de más de un recurso, puede ser definido como un texto multimodal independiente del medio -interacción cara a cara, impreso o electrónico- en el cual se distribuya (Kress $\&$ van Leeuwen, 2001).

Observar la semiosis más allá del uso de la lengua, implica aceptar que cada recurso o modo semiótico participante en un texto solo representa un significado parcial (Lemke, 1998) y que el significado completo de un texto se obtiene a partir de la interacción de la totalidad de recursos usados simultáneamente para la semiosis (O’Halloran \& Smith, 2011). Dicha interacción de varios recursos semióticos coutilizados para construir significado, es denominada intersemiosis (Jewitt, 2011).

Las investigaciones en multimodalidad de los últimos años, señalan que cada recurso semiótico ofrece un potencial para la semiosis que es incomparable con otros recursos. Este potencial semiótico se relaciona tanto con la materialidad del medio como con los usos sociales estabilizados para este medio o artefacto semiótico de acuerdo a las necesidades de cada comunidad (Kress \& van Leeuwen, 2001; Kress \& Mavers, 2005) como también con sus posibles usos futuros (Jewitt, 2011). Por ejemplo, un mapa en papel representa de manera estática la espacialidad, contigüidad, ubicación, proporciones entre elementos, a través de las convenciones de uso de recursos como colores, líneas, símbolos y lengua escrita. Dicha información interactúa para crear significado a través de la combinación de recursos descrita y difícilmente puede ser traducida solo a lengua oral o escrita manteniendo la totalidad de los significados representados originalmente en un mapa.

De esta manera, la perspectiva multimodal abre muchas interrogantes respecto a cuales son los recursos semióticos utilizados en ámbitos pedagógicos y las convenciones para significar. Entre los estudios que han abierto camino en esta línea, destaca, en primer lugar, la investigación de Jewitt, Kress, Ogborn \& Tsatsarelis (2001) sobre la retórica de la enseñanza de las ciencias en el aula, en el cual describen las explicaciones de los profesores que integran texto, dibujos y palabras. A partir de este, se encuentran aquellos sobre los gestos y el lenguaje visual utilizados en clases (Márquez et al., 2000; Pozzer-Ardenghi \& Roth, 2005), y los textos escolares desde la multimodalidad (Martins, 2006; Unsworth, 2008; Oteíza, 2009; Bezemer \& Kress, 2010), entre otros. 


\section{Marco metodológico}

La pregunta que orienta esta investigación es: ¿Cómo se despliegan las configuraciones de significado en los discursos de los profesores para enseñar historia y biología, desde una perspectiva multimodal? Interesa describir cómo participan y qué significados aportan los diferentes recursos semióticos utilizados por los profesores en los géneros para la enseñanza de la historia y de la biología.

Para alcanzar este objetivo se aborda el discurso de los profesores desde el enfoque teórico metodológico de la Semiótica Social (Jewitt, 2008; van Leeuwen, 2005). El diseño metodológico es un estudio de casos múltiples, centrado en los discursos de tres profesores de historia y tres de biología enseñando la misma unidad curricular en primer año de Educación Media. Las clases fueron grabadas y cada estudio de caso constituye un corpus de material audiovisual que se segmenta para poder explorar los patrones de significado utilizando como herramientas heurísticas las nociones de macrogénero y género curricular (Christie, 2002).

El despliegue de los significados en este registro escolar se describe desde la perspectiva multimodal (Kress, 2010), la semántica del discurso (Martin \& Rose, 2008b) y las metafunciones hallidayanas, en función de las configuraciones de significado más regulares que estructuran cada clase o género curricular. Se consideran las nociones de medio semiótico (interacción cara a cara, impresos y electrónicos), modo o recurso semiótico (lengua oral o habla, gestos, dibujos, fotos, escritura, etc.). En este estudio no se profundiza en la prosodia.

\section{Hallazgos}

A continuación se presentan los principales géneros para la enseñanza identificados en los discursos de los profesores en clases de historia y luego de biología. Cada uno de ellos será descrito poniendo el foco en cómo los distintos recursos semióticos contribuyen a la construcción de significado ideacional propio de cada disciplina en el discurso del profesor.

\subsection{Recursos semióticos para la representación de la causalidad, el tiempo y el espacio en el discurso de la enseñanza de la historia}

En relación con el discurso del profesor en clases de historia enseñando la unidad curricular 'Segunda Guerra Mundial', encontramos dos principales configuraciones de significado que son instanciadas regularmente en los macrogéneros o unidades didácticas en estudio: género explicación de las causas (explicación factorial) y género relato histórico. 
Tal como se puede ver en la Tabla 1, el género explicación de las causas se despliega en tres etapas: 1) exposición del evento; 2) dinámica de preguntas y respuestas en torno a las causas y; 3 ) síntesis de las causas.

Tabla 1. Etapas genéricas de explicación de las causas en la enseñanza de la historia.

\begin{tabular}{|c|c|c|c|}
\hline Etapas & $\begin{array}{l}\text { 1) Exposición del } \\
\text { evento }\end{array}$ & $\begin{array}{l}\text { 2) Dinámica de preguntas y respuestas } \\
\text { en torno a las causas }\end{array}$ & 3) Síntesis de las causas \\
\hline $\begin{array}{l}\text { Medio y } \\
\text { recursos } \\
\text { semióticos }\end{array}$ & $\begin{array}{l}\text { INTERACCIÓN } \\
\text { CARA A CARA: } \\
\text { Lengua oral/gestos }\end{array}$ & $\begin{array}{l}\text { INTERACCIÓN CARA A CARA/ } \\
\text { PIZARRÓN/PRESENTACIÓN PPT: } \\
\text { Lengua oral/gestos/dibujo/escritura }\end{array}$ & $\begin{array}{c}\text { INTERACCIÓN CARA A } \\
\text { CARA: } \\
\text { Lengua oral/gestos }\end{array}$ \\
\hline
\end{tabular}

El recurso semiótico que articula este género es la lengua oral desplegada por el profesor en la interacción cara a cara con sus estudiantes. La lengua oral posibilita tanto la construcción interactiva del significado ideacional de la historia -en una dinámica de preguntas y respuestas- así como del significado interpersonal. En relación al significado interpersonal, el profesor presupone la causalidad como la interpretación compartida con los estudiantes acerca de los eventos históricos, enseñándoles a asumir que los hechos siempre tienen causas o factores, tal como se hace en historia.

Es importante relevar que la dinámica de preguntas y respuestas entre cada uno de los profesores y sus estudiantes es central en este género, ya que favorece un movimiento entre el conocimiento fuera de sentido común y el de sentido común. El primero es aportado por el profesor, quien usa términos técnicos y empaqueta los conceptos en metáforas gramaticales o nominalizaciones (Halliday \& Martin, 1992) en base a las respuestas de sus estudiantes expresadas en una gramática congruente.

Profesor: El eje, chicos, va a ser el grupo de países que está conformado por Alemania, Italia y Japón... ¿Japón ...porque se integra a este grupo? una respuesta tremendamente fácil y sencilla

Alumno: porque está en Asia

Profesor: no, no es una respuesta geográfica, es una respuesta ideológica

Alumno: es porque está en contra de Estados Unidos

Profesor: ya, por lo tanto ¿simpatía por qué ideología tienen? ... Por el nacismo y fascismo, ya? Este es un grupo. Pregunto: ¿Cual será el otro grupo que se opone?

Alumno: los aliados

En el extracto del diálogo se puede apreciar cómo el profesor introduce conceptos técnicos (en negrita) en un juego lingüístico en el cual los países son los participantes de procesos existenciales y materiales. El profesor en interacción con los estudiantes construye taxonomías técnicas como eje/aliados, factores geográficos/ideológicos, 
ideología: nacismo/facismo. Es justamente en esta etapa en la que se puede apreciar que los profesores recurren a otros recursos semióticos para significar el establecimiento de las causas.

De acuerdo a lo observado, hay dos casos en los cuales el profesor de historia recurre al pizarrón y a la presentación computacional (Power Point) de manera entrelazada a la interacción cara a cara. En el primer caso, la lengua oral construye un significado de manera entrelazada con la escritura en el pizarrón y los gestos sobre ella, para la reinterpretación de la experiencia en una versión gramaticalmente incongruente o metafórica. En este paso parece ser muy importante que mientras 'traduce' la versión de los estudiantes, escribe o señala la escritura de los nombres abstractos y técnicos, así como las nominalizaciones que constituyen la versión esperada de las causas (ver Foto 1).

Si se pone atención a la expresión de la escritura, esta es usada aquí de una manera particular configurando un listado o punteo que concentra cada significado técnico en grupos nominales. De esta manera, quedan impresas sobre el pizarrón expresiones como: crisis económica, nazismo, fascismo, exceso de diplomacia, entre otras. Algo similar ocurre con el uso de la presentación computacional, la cual despliega para los estudiantes conceptos como: fracaso de Tratado de Versalles, rivalidad ideológica, eje Roma, Berlín, Tokio; etc. (Foto 1), muchas de ellas, metáforas gramaticales.

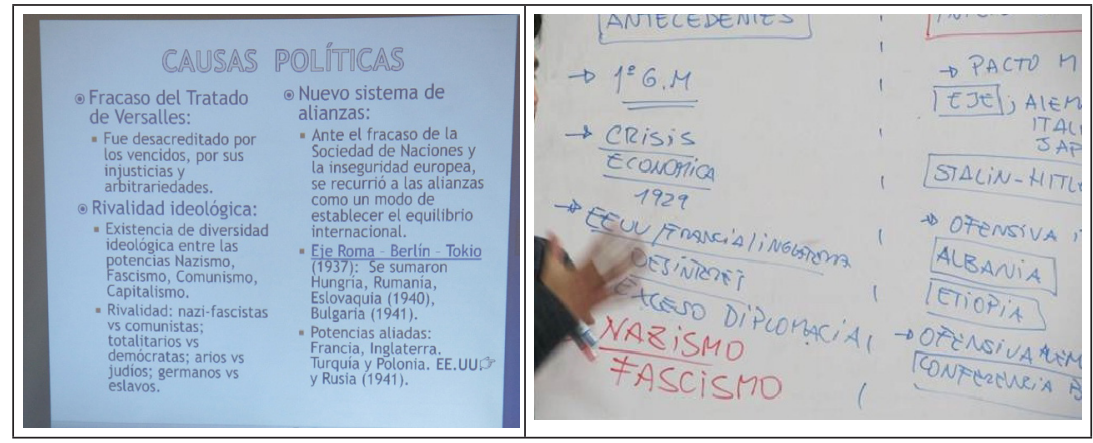

Foto 1. Versión fuera de sentido común, habla, gestos y escritura.

La combinación semiótica habla/gestos/escritura permite al profesor focalizar en los nombres de conceptos científicos y su denominación técnica. Frecuentemente, la denominación técnica y las taxonomías se construyen de manera intersemiótica (Manghi, 2010), por ejemplo el profesor dice 'los aliados', construyendo la categoría mediante la lengua oral, mientras muestra mediante un gesto indexical las palabras escritas que aluden a los miembros de esa categoría (EEUU/Francia/Inglaterra) (Foto 1).

El segundo caso corresponde a la definición de un concepto espacial, como por ejemplo 'frente de batalla', en el cual el profesor dibuja en el pizarrón un círculo 
y luego mediante flechas y escritura construye de manera entrelazada con gestos y lengua oral una categoría y sus subtipos: Frente de batalla: frente oriental/frente occidental (ver Tabla 2). El profesor usa la lengua oral para establecer los nombres de los tres conceptos técnicos y los presenta como una tipología especializada para el discurso de la historia, mientras gestos y escritura sobre el pizarrón indican posiciones y direccionalidad en torno a un círculo central, construyendo el significado nuclear de esta noción espacial.

Tabla 2. Ideación intersemiótica: Frentes de batalla.

\begin{tabular}{|l|l|}
\hline P: ALEMANIA, TUVO QUE \\
ENFRENTARSE (dibuja círculo y dos flechas a \\
cada lado) \\
\hline P: EN DOS FRENTES \\
\hline $\begin{array}{l}\text { P: UN FRENTE ORIENTAL (escribe en el } \\
\text { pizarrón FRENTE ORIENTAL) }\end{array}$ \\
\hline $\begin{array}{l}\text { P: Y UN FRENTE OCCIDENTAL (escribe } \\
\text { FRENTE OCCIDENTAL) }\end{array}$ \\
\hline
\end{tabular}

Respecto del segundo género que articula el despliegue del macrogénero curricular en clases de historia, este corresponde al relato histórico. Tal como se ve en la Tabla 3 , las etapas que constituyen este género son las siguientes: 1) presentación del contexto histórico y 2) secuenciación de eventos y, de manera incrustada se encuentra la evaluación del evento histórico.

Tabla 3. Etapas genéricas del recuento histórico, en la enseñanza de la historia.

\begin{tabular}{|c|c|c|c|}
\hline Etapas & $\begin{array}{l}\text { 1) Presentación del con- } \\
\text { texto histórico }\end{array}$ & 2) Secuenciación de eventos & $\begin{array}{l}\text { (-) Evaluación del } \\
\text { evento }\end{array}$ \\
\hline $\begin{array}{l}\text { Medio y } \\
\text { recursos } \\
\text { semióticos }\end{array}$ & $\begin{array}{l}\text { INTERACCIÓN CARA A } \\
\text { CARA: } \\
\text { Lengua oral/gestos }\end{array}$ & $\begin{array}{l}\text { INTERACCIÓN CARA A CARA/ } \\
\text { PIZARRÓN/MAPA: } \\
\text { Lengua oral/gestos/dibujo/escritura }\end{array}$ & $\begin{array}{l}\text { INTERACCIÓN } \\
\text { CARA A CARA } \\
\text { Lengua oral/gestos }\end{array}$ \\
\hline
\end{tabular}

El recuento histórico se despliega entrelazando conocimiento temporal con espacial. La interacción cara a cara nuevamente estructura este despliegue en torno a la lengua oral, pero en este caso, mapa y pizarrón aportan gran parte de la carga semiótica en la etapa central del género: secuenciación de los eventos.

La secuenciación de los eventos históricos es llevada a cabo por los profesores de manera gramaticalmente congruente, presentando a los países y sus líderes en acciones y comportamientos. Juegan un rol muy importante en este relato la representación de las circunstancias temporales. El medio común utilizado es el pizarrón para desplegar de manera entrelazada con la lengua oral, representaciones visuales de convenciones temporales. 


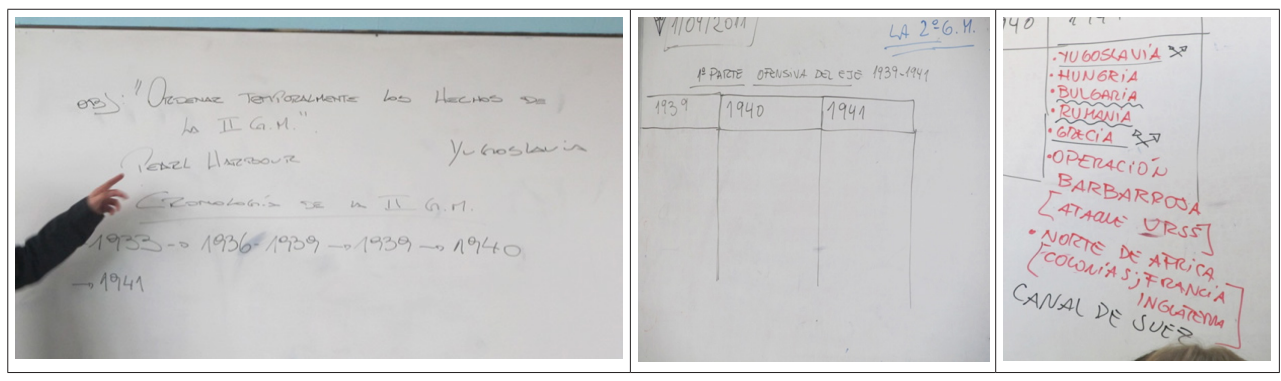

Foto 2. Representaciones de convenciones para representar el tiempo visualmente.

La Foto 2 muestra las representaciones en el pizarrón de la línea de tiempo así como de la tabla cuyas columnas ordenan temporalmente la información y al interior de cada columna vuelven a presentar verticalmente la información en orden temporal. Estas representaciones ayudan no solo a construir la secuencia de los eventos en el tiempo, sino que sirven además a la función textual. La construcción progresiva de la representación temporal en el pizarrón da un ritmo particular al despliegue del género en la interacción cara a cara, marcando fases en la construcción del evento completo.

El espacio en este género, se representa de manera entretejida con el tiempo. Los profesores usan distintas estrategias para textualizar los cambios espaciales en el tiempo. Por ejemplo, una de las opciones de los profesores es entrelazar gestos deícticos sobre un mapa, los cuales, junto con la lengua oral, dan cuenta de una secuencia de eventos.

Tal como se ve en la Foto 3, otra opción tomada por un profesor es no usar un mapa sino el medio semiótico pizarrón para realizar un complejo patrón de significados en los cuales construye el relato mediante la lengua oral, el dibujo de un mapa y de una tabla. Mediante el habla o lengua oral despliega los eventos y simultáneamente va modificando el dibujo del mapa para representar los países que ya se han definido lingüísticamente, ahora de manera topológica y, a continuación, escribe el nombre del país en la tabla, en la columna de la fecha que corresponde. El achurado progresivo sobre el dibujo del mapa complementa la información lingüística con las circunstancias espaciales que anclan estos eventos empaquetados como nombres y que dan cuenta de los rasgos espaciales del sucesivo avance militar. 


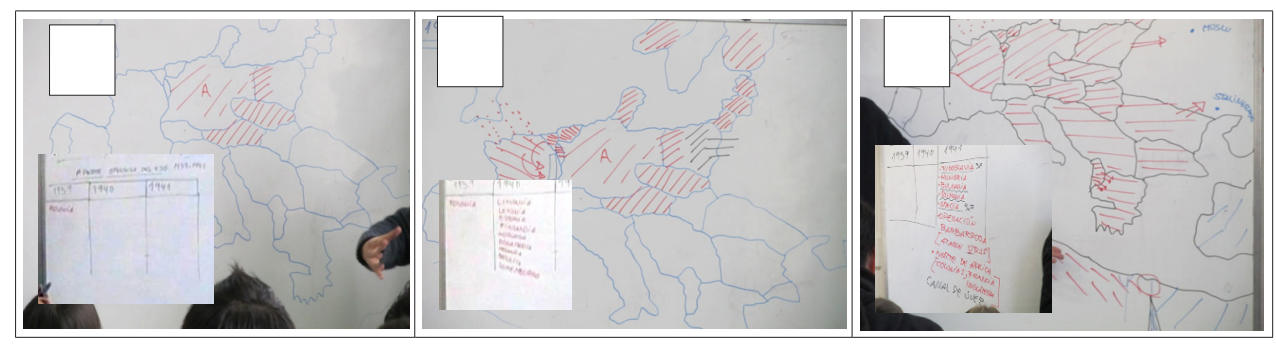

Foto 3. Tres momentos del despliegue tiempo/espacio.

Dichos patrones de significados ilustran la forma como el profesor construye el tiempo y el espacio de manera intersemiótica en el género relato histórico, mediante un uso importante del recurso visual mapa. Para construir el concepto de país, al mismo tiempo que entregar el nombre y presentarlo como agente, el profesor enriquece visualmente la noción territorial que implica considerar su ubicación espacial respecto de los países vecinos, así como la extensión del territorio, sus límites y proporciones en la representación convencional del mapa. De esta manera, se configura la taxonomía categoría/miembro, como países que participan en la guerra y cada miembro de esta categoría es representado con sus propiedades geográficas así como políticas, constituyendo además la taxonomía espacial partes/todo.

La Tabla 4 resume los hallazgos para los estudios de caso de clases de historia en cuanto a los géneros en el registro escolar, sus funciones para la enseñanza así como las combinaciones de medios y recursos semióticos utilizados por el profesor para representar el significado de manera intersemiótica.

Tabla 4. Síntesis de géneros, funciones y recursos semióticos en la enseñanza de la historia.

\begin{tabular}{|l|l|l|l|l|}
\hline Propósito Social & $\begin{array}{l}\text { Género/ registro } \\
\text { Escolar }\end{array}$ & $\begin{array}{l}\text { Función } \\
\text { pedagógica }\end{array}$ & Tipos de género & $\begin{array}{l}\text { Combinación semiótica } \\
\text { Medio-(recursos) }\end{array}$ \\
\hline $\begin{array}{l}\text { Explicar cómo } \\
\text { ocurren los } \\
\text { eventos }\end{array}$ & $\begin{array}{l}\text { Explicación en la } \\
\text { enseñanza de la } \\
\text { historia }\end{array}$ & $\begin{array}{l}\text { Enseñar a pensar y } \\
\text { representar la historia } \\
\text { desde la causalidad }\end{array}$ & $\begin{array}{l}\text { Explicación factorial: } \\
\text { Secuencia simple de } \\
\text { causas que explican un } \\
\text { evento histórico }\end{array}$ & $\begin{array}{l}\text { Interacción cara a cara (habla } \\
\text { y gestos)/pizarrón (dibujo } \\
\text { computacional(escritura) }\end{array}$ \\
\hline $\begin{array}{l}\text { Secuenciar } \\
\text { eventos }\end{array}$ & $\begin{array}{l}\text { Relato histórico en } \\
\text { la enseñanza de la } \\
\text { historia }\end{array}$ & $\begin{array}{l}\text { Enseñar a anclar } \\
\text { eventos históricos en } \\
\text { el tiempo y espacio } \\
\text { para describir un } \\
\text { proceso }\end{array}$ & $\begin{array}{l}\text { Recuato histórico: } \\
\text { Seventos históricos en } \\
\text { el tiempo y espacio } \\
\text { geográfico }\end{array}$ & $\begin{array}{l}\text { Interacción cara a cara } \\
\text { (habla y gestos)/pizarrón } \\
\text { (dibujo y tabla escrita) o } \\
\text { mapa (convenciones color y } \\
\text { simbología) }\end{array}$ \\
\hline
\end{tabular}




\subsection{Recursos semióticos para la representación de las definiciones y las consecuencias en el discurso de la enseñanza de la biología}

Los profesores de biología observados enseñando la unidad 'Nutrición celular', despliegan dos géneros principales: la definición (o informe según la LSF) y la explicación secuencial.

Respecto del primero de ellos: la definición, esta se despliega en las siguientes etapas genéricas: 1) pregunta por concepto/por tipología (tipos o categorías)/ por componentes (partes); 2) dinámica de preguntas y respuestas para construir la definición y; 3) definición final.

Tabla 5. Etapas genéricas de la definición en el discurso de la enseñanza de biología.

\begin{tabular}{|l|l|l|l|}
\hline Etapas & $\begin{array}{l}\text { 1) Pregunta por } \\
\text { concepto/tipo/parte }\end{array}$ & $\begin{array}{l}\text { 2) Dinámica de preguntas y respuestas } \\
\text { en torno a la definición }\end{array}$ & 3) Definición final \\
\hline $\begin{array}{l}\text { Medio y } \\
\text { recursos } \\
\text { semióticos }\end{array}$ & $\begin{array}{l}\text { INTERACCIÓN CARA } \\
\text { A CARA: } \\
\text { Lengua oral/gestos }\end{array}$ & $\begin{array}{l}\text { INTERACCIÓN CARA A CARA/ } \\
\text { PIZARRÓN/PRESENTACIÓN PPT: } \\
\text { Lengua oral/gestos/dibujo/escritura }\end{array}$ & $\begin{array}{l}\text { INTERACCIÓN CARA } \\
\text { A CARA: } \\
\text { Lengua oral/gestos }\end{array}$ \\
\hline
\end{tabular}

En las clases de biología se identificaron tres tipos de definiciones: descriptivas, clasificatorias y composicionales (Martin \& Rose, 2008a). En todas ellas los profesores utilizan el pizarrón y de manera secundaria algunos de ellos la presentación computacional para construir la etapa central del género, en la que mediante preguntas (¿qué es la osmosis?/¿qué tipos de proteínas hay?/¿qué componentes tiene la membrana plasmática?) y la reformulación de las respuestas de los aprendices, los acercan a la definición científica del mundo natural mediante una versión de la experiencia gramaticalmente incongruente.

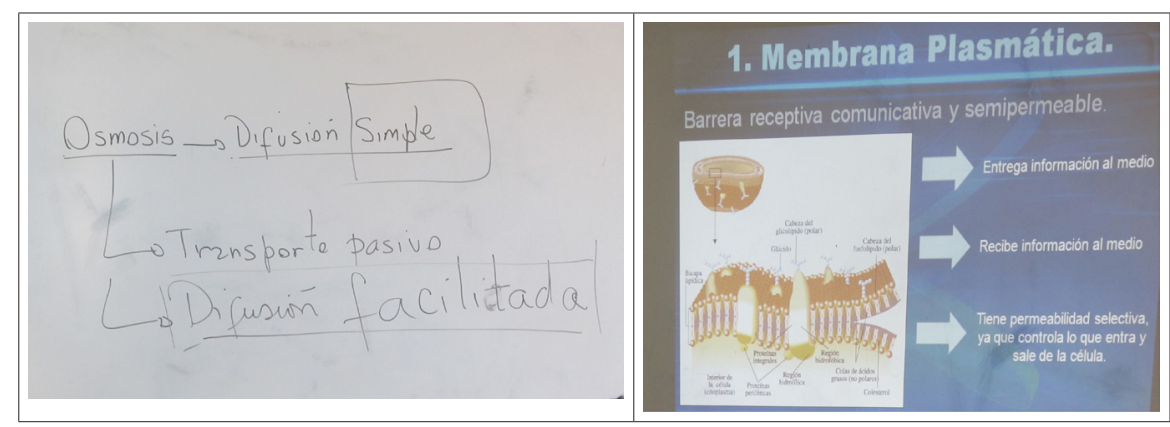

Foto 4. Medios y recursos semióticos en la definición descriptiva.

En relación con las definiciones descriptivas, la etapa nuclear construye las distintas características del concepto a definir, como osmosis o membrana plasmática. Cada profesor, mediante la escritura y las flechas u otro marcador en la pizarra o en 
la presentación computacional, hacen más visible para los estudiantes distintos rasgos técnicos que definen el concepto (Foto 4). Los profesores recurren reiteradamente a apuntar estos conceptos escritos durante la etapa central y final del despliegue de la definición, en el seguimiento de los participantes en los procesos atributivos y relacionales, y sus propiedades.
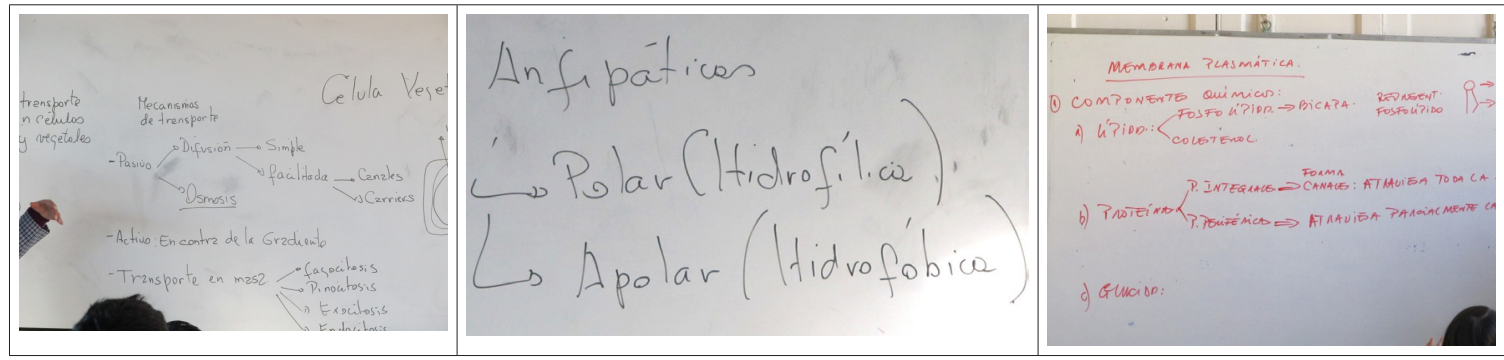

Foto 5. Definiciones clasificatorias escritas en enseñanza de la biología.

En las definiciones clasificatorias (Foto 5) la pizarra cumple un rol esencial, ya que los profesores construyen gradualmente las clasificaciones mediante la lengua oral y, al mismo tiempo, escriben en la pizarra grupos nominales que explicitan las tipologías (componentes químicos: lípidos, proteínas, glúcidos), conceptos opuestos (polar/apolar) y categorías anidadas (transporte activo/pasivo: osmosis/difusión: simple/facilitada). Las clasificaciones en el pizarrón visibilizan las jerarquías y relaciones semánticas entre conceptos técnicos y especializados, explicitando para los estudiantes la organización taxonómica de los conceptos científicos.

Con respecto a las definiciones composicionales, tal como se ve en la Foto 6, en ellas los dibujos de esquemas anatómicos juegan un papel imprescindible. Estas representaciones visuales son además etiquetadas lingüísticamente y mostradas por los profesores para explicar las partes que componen diversas estructuras. Lo que la lengua oral construye como una relación todo/ partes, los dibujos modelan como una propuesta analítica de la estructura total, el tamaño, forma, posición y proporción entre elementos de un todo.
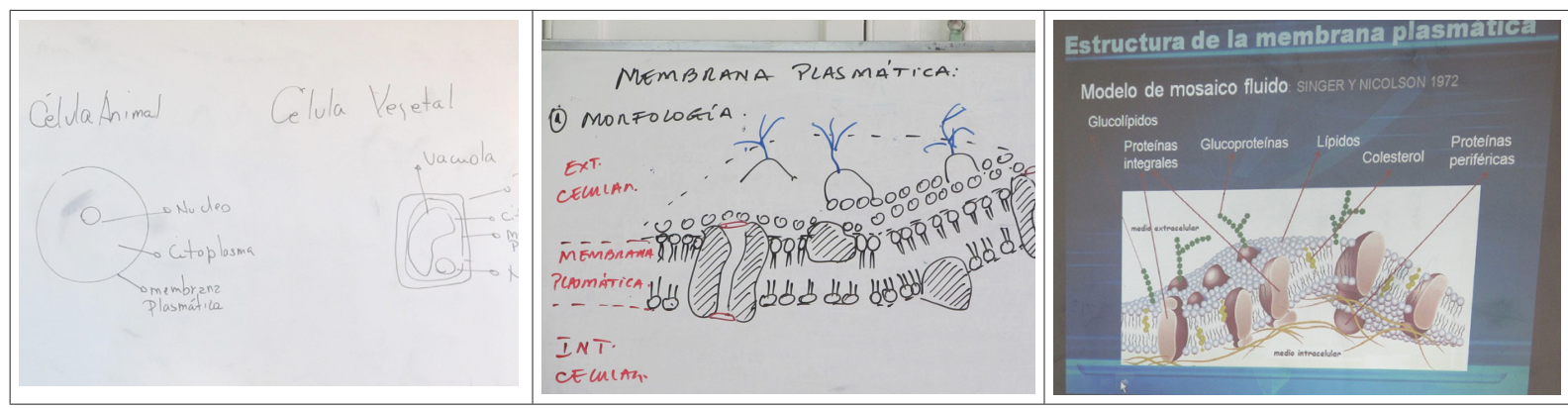

Foto 6. Dibujos esquemáticos en la definición composicional. 
Las definiciones composicionales visuales se vuelven fundamentales en el despliegue del macrogénero curricular, ya que los profesores las usan para apoyar tanto las definiciones clasificatorias, por ejemplo para la construcción de la diferencia entre proteínas integrales y proteínas periféricas que los profesores levantan sobre los dibujos de esquemas mediante gestos y lengua oral, así como también para el segundo género: las explicaciones.

Las explicaciones secuenciales son desplegadas por los profesores en tres fases: 1) asociación a ejemplo cotidiano; 2) explicación del proceso y; 3) síntesis del proceso.

Tabla 6. Etapas genéricas de la explicación, en el discurso de la enseñanza de biología.

\begin{tabular}{|c|c|c|c|}
\hline Etapas & $\begin{array}{l}\text { 1) Asociación a ejemplo } \\
\text { cotidiano }\end{array}$ & 2) Explicación del proceso & 3) Síntesis del proceso \\
\hline $\begin{array}{l}\text { Medio y } \\
\text { recursos } \\
\text { semióticos }\end{array}$ & $\begin{array}{l}\text { INTERACCIÓN CARA } \\
\text { A CARA/ PIZARRÓN: } \\
\text { Lengua oral/gestos/ } \\
\text { dibujo }\end{array}$ & $\begin{array}{l}\text { INTERACCIÓN CARA } \\
\text { A CARA/ PIZARRÓN/ } \\
\text { PRESENTACIÓN PPT: } \\
\text { Lengua oral/gestos/dibujo/ } \\
\text { escritura }\end{array}$ & $\begin{array}{l}\text { INTERACCIÓN CARA } \\
\text { A CARA/PIZARRÓN / } \\
\text { PRESENTACIÓN PPT: } \\
\text { Lengua oral/gestos/dibujo }\end{array}$ \\
\hline
\end{tabular}

En la primera parte de la explicación, los profesores utilizan ejemplos de la vida cotidiana para proponer las explicaciones científicas sobre los procesos naturales. De esta manera, mediante la lengua oral y gestos, y además dibujos, proponen situaciones conocidas a los aprendices como una pieza cuyo muro se inunda, una flor que se marchita o entrar y salir del estadio.

Luego de esta situación descrita con los aportes de los estudiantes, los profesores se vuelven hacia los procesos naturales para basarse en representaciones de esquemas anatómicos y dinamizarlos a través de flechas, de la modificación de los dibujos o de los gestos sobre ellos, junto con la explicación lingüística (Foto 7).
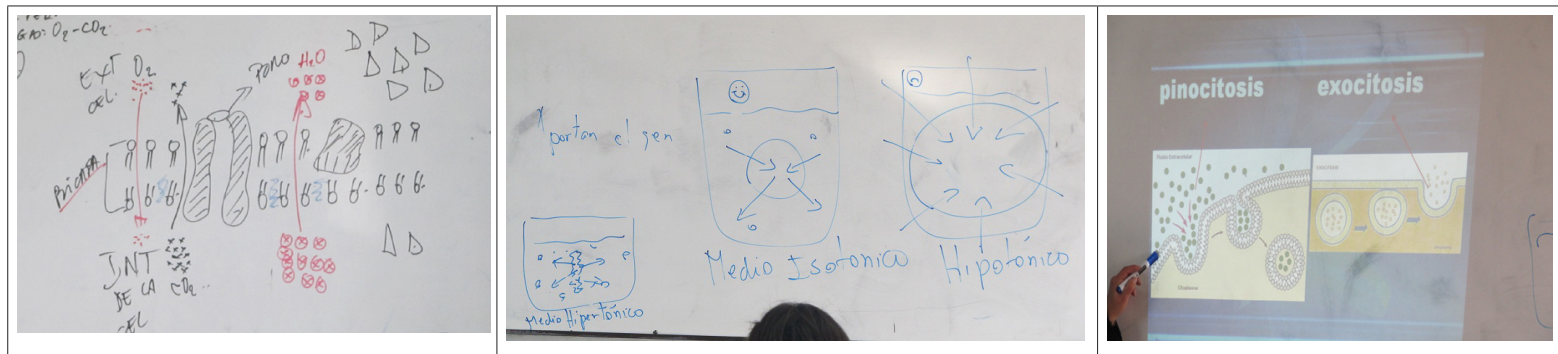

Foto 7. Dinamización de dibujos para la explicación de las consecuencias.

Los mismos dibujos que primero servían a la definición luego, con la mediación del profesor a través de la lengua oral y gestos sobre los esquemas, se transforman en recursos para explicar diferentes momentos de un proceso natural y la manera como las estructuras anatómicas se modifican en dicho proceso. 
La Tabla 7 resume los géneros en el registro escolar en las clases de biología estudiadas, sus funciones para la enseñanza así como las combinaciones de medios y recursos semióticos utilizados por los profesores.

Tabla 7. Síntesis géneros, funciones y recursos semióticos en la enseñanza de la biología.

\begin{tabular}{|c|c|c|c|c|}
\hline $\begin{array}{l}\text { Propósito } \\
\text { Social }\end{array}$ & $\begin{array}{l}\text { Género/ } \\
\text { registro escolar }\end{array}$ & $\begin{array}{l}\text { Función } \\
\text { pedagógica }\end{array}$ & Tipos de género & $\begin{array}{l}\text { Combinación semiótica } \\
\text { Medios y (recursos) }\end{array}$ \\
\hline $\begin{array}{l}\text { Informar } \\
\text { sobre cómo } \\
\text { se entiende el } \\
\text { mundo } \\
\text { o Definir }\end{array}$ & $\begin{array}{l}\text { Definición en la } \\
\text { enseñanza de la } \\
\text { biología }\end{array}$ & $\begin{array}{l}\text { Enseñar a describir, } \\
\text { clasificar y componer } \\
\text { objetos y/o } \\
\text { fenómenos del mundo } \\
\text { natural desde la } \\
\text { perspectiva científica }\end{array}$ & $\begin{array}{l}\text { Definición descriptiva: } \\
\text { Describe el fenómeno en } \\
\text { función de sus rasgos. } \\
\text { Definición clasificatoria: } \\
\text { Sub-clasifica fenómenos con } \\
\text { respecto a criterios dados. } \\
\text { Definición composicional: } \\
\text { Describe los componentes de } \\
\text { una entidad. }\end{array}$ & $\begin{array}{l}\text { Medio Interacción cara } \\
\text { a cara (lengua oral y } \\
\text { gestos)/ pizarrón (dibujos } \\
\text { y escritura)/ presentación } \\
\text { computacional (esquemas y } \\
\text { escritura) }\end{array}$ \\
\hline $\begin{array}{l}\text { Explicar } \\
\text { como ocurren } \\
\text { procesos }\end{array}$ & $\begin{array}{l}\text { Explicación en la } \\
\text { enseñanza de la } \\
\text { biología }\end{array}$ & $\begin{array}{l}\text { Enseñar a pensar en } \\
\text { términos de causa y } \\
\text { efecto para explicar } \\
\text { procesos naturales } \\
\text { desde la perspectiva } \\
\text { científica }\end{array}$ & $\begin{array}{l}\text { Explicación secuencial: } \\
\text { Secuencia simple de causas y } \\
\text { efectos. }\end{array}$ & $\begin{array}{l}\text { Medio Interacción cara } \\
\text { a cara (lengua oral y } \\
\text { gestos)/ pizarrón (dibujos } \\
\text { y escritura)/ pt. comp. } \\
\text { (esquemas y escritura) }\end{array}$ \\
\hline
\end{tabular}

\section{CONCLUSIONES}

El enfoque adoptado por este estudio permite preguntar por el rol de los diversos recursos en la construcción de los significados en el discurso del profesor y focalizar en la semiosis según las distintas opciones del docente de acuerdo a las condiciones sociales y materiales de cada contexto educativo.

Si bien el habla o lengua oral del profesor sin duda estructura los géneros descritos, también comparten la carga semiótica otros recursos que son entretejidos en el discurso de los profesores para construir el significado mediante la interacción de todos ellos. La escritura como listado o punteo, despliega de manera nuclear los conceptos técnicos y las metáforas gramaticales, representando de manera visible la organización taxonómica de los conceptos propios de las dos disciplinas. En el discurso de la enseñanza, la escritura desplegada en conjunto con la lengua oral por el profesor focaliza en las denominaciones lingüísticas de conceptos científicos que deben ser aprendidos por los estudiantes. En cuanto a otros recursos como los mapas para la historia y los dibujos esquemáticos para la biología, estos favorecen la representación convergente de conocimiento temporal y espacial. Por una parte, para la historia, los gestos y habla junto con los mapas permiten, el despliegue entrelazado de eventos históricos en el espacio geográfico para el relato histórico. Por otra parte, en biología, los esquemas anatómicos participan de manera importante en las definiciones composicionales. Estos mismos son dinamizados mediante la lengua 
oral, gestos y dibujos de flechas representando distintos momentos de un proceso a medida que la estructura se modifica o cambia de posición, en las explicaciones.

En esta descripción de los géneros desde la perspectiva multimodal, pizarrón y presentación computacional en la enseñanza cara a cara aportan de manera importante al andamiaje que lleva a cabo el profesor, favoreciendo el movimiento entre las dos versiones gramaticales -congruente e incongruente- del evento histórico o del proceso natural. Al mismo tiempo, el despliegue de dibujos, mapas, esquemas, líneas de tiempo y otros, cumple una función para la alfabetización semiótica. El profesor además de relatar la historia o definir procesos naturales, introduce a los estudiantes en convenciones sobre cómo representar e interpretar el tiempo, el espacio, así como las secuencias por medio de recursos visuales.

A partir del discurso de los profesores de este estudio se ha podido identificar cómo a través de las etapas genéricas se produce una mediación semiótica sobre las formas de representar y comunicar el conocimiento de la disciplina para los estudiantes. Cabe preguntarse qué falta hacer para favorecer el aprendizaje de estas formas de pensar y comunicar que se alejan de lo cotidiano. Según la propuesta de la Escuela de Sydney, el movimiento sucesivo entre el empaquetamiento del conocimiento en formas gramaticales incongruentes y desempaquetamiento en formas gramaticalmente congruentes debería favorecer el aprendizaje de los discursos de las disciplinas a los estudiantes. Los profesores observados realizan dicho movimiento semántico, sin embargo, es posible reconocer dos oportunidades de mejora para estas prácticas pedagógicas. La primera tiene que ver con diseñar momentos para que los estudiantes puedan expresarse de manera oral y escrita tal como se espera que lo hagan en historia o en biología antes de ser evaluados, para que puedan ejercitar la forma de empaquetar el conocimiento y construir un discurso metafórico. La segunda oportunidad, se relaciona con ir más allá de evaluaciones finales centradas en pruebas de selección múltiple, ejercitando y evaluando también la apropiación de los potenciales para significar a través de las distintas representaciones propias de las disciplinas.

Finalmente, es necesario destacar el aporte de perspectiva multimodal a la lingüística educacional, ya que aparte de plantear nuevas preguntas y abordajes de análisis a las prácticas pedagógicas, permite la comprensión del uso de la lengua en su contexto semiótico y social. 


\section{REFERENCIAS BIBLIOGRÁFICAS}

Barletta, N. \& Mizuno, J. (2011). Una propuesta metodológica para la meta reconstrucción histórica. En T. Oteíza \& D. Pinto (Eds.), En (re)construcción: Discurso, identidady nación en los manuales escolares de historia y de ciencias sociales (pp. 89-128). Santiago: Cuarto Propio.

Bernstein, B. (1990). Class, codes and control vol 4: The structuring of pedagogic discourse. Londres: Routledge.

Bernstein, B. (2000). Pedagogy, symbolic control and identity. Londres: Rowan y Littlefield.

Bezemer, J. \& Kress, G. (2010). Changing text: A social semiotic analysis of textbooks. Design for Learning, 3(1-2), 10-29.

Christie, F. (2002). Classroom discourse analysis: A functional perspective. Londres: Continuum.

Christie, F. \& Derewianka, B. (2010). School discourse. Londres: Continuum.

Coffin, C. (1997). Constructing and giving value to the past: An investigation into Secondary School History. En F. Christie \&J. Martin (Eds.), Genre and institutions: Social processes in the work place and school (pp. 196-230). Londres: Cassell.

Eggins, S. \& Martin, J. (2003). El contexto como género: Una perspectiva lingüística funcional. Revista Signos. Estudios de Lingüística, 36(54), 185-205.

Giudice, J. \& Moyano, E. (2011). Género y formación de ciudadanos: La reconstrucción del periodo 1976-1983 en manuales argentinos para la escuela primaria. En T. Oteíza \& D. Pinto (Eds.), En (re)construcción: Discurso, identidady nación en los manuales escolares de bistoria y de ciencias sociales (pp. 205-268). Santiago: Cuarto Propio.

Halliday, M. (1982). El lenguaje como semiótica social. México, D.F.: Fondo de Cultura Económica.

Halliday, M. \& Martin, J. (1993). Writing science: Literacy and discursive power. Pittsburgh: University of Pittsburgh.

Hodge, R. \& Kress, G. (1988). Social Semiotics. Cambridge: Polity.

Iedema, R. (2003). Multimodality, resemiotization: Extending the analysis of discourse as multisemiotic practice. Visual Communication, 2(1), 29-58.

Jewitt, C. (2008). Multimodal classroom research. AERA Review of Research in Education, 32, 241-267. 
Jewitt, C. (2011). The changing pedagogic landscape of subject English in UK classrooms. En K. O’Halloran \& B. Smith (Eds.), Multimodal studies: Exploring issues and domains (pp.184-201). Nueva York: Routledge.

Jewitt, C., Kress, G., Ogborn, J. \& Tsatsarelis, C. (2001). Multimodal teaching and learning: The rhetorics of the classroom. Londres: Continuum.

Kress. G. \& Mavers, D. (2005). Social semiotics and multimodal texts. En B. Somehk \& C. Lewin (Eds.), Research methods in the social science (pp. 172-193). Londres: Sage.

Kress, G. (2010). Multimodality: A social semiotic approach to contemporary communication. Londres: Routledge.

Kress, G. \& van Leeuwen, T. (2001). Multimodal discourse. The modes and media of contemporary communication. Londres: Arnold.

Latour, B. (2008). Reensamblar lo social: Una introducción a la teoría del actor-red. Buenos Aires: Editorial Manantial.

Lemke, J. (1998). Multiplying meaning: Visual and verbal semiotics in scientific text. En J. Martin \& R. Veel (Eds.), Reading science: Critical and functional perspectives on discourses of science (pp. 87-113). Londres: Routledge.

Manghi, D. (2010). Recursos semióticos del profesor de matemática: Funciones complementarias del habla y los gestos para la alfabetización científica escolar. Estudios Pedagógicos, XXXVI(2), 99-115.

Malinowsky, B. (1933). Coral gardens and their magic. Londres: Goerge Allen y Edwin.

Márquez, C., Izquierdo, M. \& Espinet, M. (2000). Comunicación multimodal en la clase de ciencias: El ciclo del agua. Enseñanza de las ciencias, 21(3), 371-386.

Martin, J. (2003). Making history, grammar for interpretation. En J. Martin \& R. Wodak (Eds.), Re/reading the past: critical and functional perspectives on time and value (pp. 19-57). Amsterdam: Benjamins.

Martin, J. \& Rose, D. (2008a). Genre relations: Mapping culture. Londres: Equinox.

Martin, J. \& Rose, D. (2008b). Working with discourse: Meaning beyond the clause. Londres: Continuum.

Martin, J., Maton, K. \& Matruglio, E. (2010). Historical cosmologies: Epistemology and axiology in Australian secondary school history discourse. Revista Signos. Estudios de Lingüística, 43(74), 433-463. 
Martins, I. (2006). Analisando livros didáticos na perspectiva dos Estudos do Discurso: Compartilhando reflexões e sugerindo uma agenda para a pesquisa. Pro-Posições, 7(1), 49.

Maton, K. (2007). Knowledge-knower structures in intellectual and educational fields. En F. Christie \& J. Martin (Eds.), Language, knowledge and pedagogy: functional linguistics and sociological perspective (pp. 87-108). Londres: Continuum.

Moyano, E. (2010). Aportes del análisis de género y discurso a los procesos de enseñanza y aprendizaje escolares: Las ciencias biológicas y la historia. Discurso \& Sociedad, 4(2), 294-331.

Moss, G. \& Chamorro, D. (2011). La enseñanza de las ciencias sin asidero en el tiempo ni el espacio: Análisis del discurso de dos textos escolares. En N. Barletta \& D. Chamorro (Eds.), Texto escolar y aprendizaje (pp. 123-148). Barranquilla: Universidad del Norte.

O'Halloran, K. \& Smith, B. (Eds.). (2011). Multimodal studies: Exploring issues and domains. Nueva York: Routledge.

Oteíza, T. (2006). Discurso pedagógico de la bistoria. Un análisis lingüístico sobre construcción ideológica de la bistoria de Chile (1970-2001). Santiago: Frasis.

Oteíza, T. (2009). Diálogo entre textos e imágenes: Análisis multimodal de textos escolares desde una perspectiva intertextual. Delta, 25, 657-664.

Oteíza, T. \& Pinto, D. (2011). En (Re) construcción discurso, identidady nación en los manuales escolares de historia y de ciencias sociales. Santiago: Cuarto Propio.

Parodi, G. (2010). Multisemiosis y Lingüística de Corpus: Artefactos (multi) semióticos en los textos de seis disciplinas en el corpus Pucv-2010. Revista de Lingüistica Aplicada, 48(2), 33-70.

Pozzer-Ardenghi, P. \& Roth, W. (2005). Photographs in lectures: Gestures as meaning-making resources. Linguistics and Education, 15, 275-293.

The New London Group (1996). A pedagogy of multiliteracies: Designing social futures. Harvard Educational Review, 66(1), 60-92.

Unsworth, L. (Ed.). (2008). Multimodal semiotics and multiliteracies education: Transdisciplinary approaches to research and professional practice. Londres: Continuum.

Van Leeuwen, T. (2005). Introducing social semiotics. Londres: Routledge.

Veel, R. (1998). The greening of school science: Ecogenesis in secondary classrooms. En J. Martin \& R. Veel (Eds.), Reading science. Critical and functional perspectives on discourses of science (pp. 114-151). Londres: Routledge. 